\title{
Comparative analysis of ESTs involved in grape responses to Xylella fastidiosa infection Hong Lin* ${ }^{*}$, Harshavardhan Doddapaneni ${ }^{\dagger 1,2}$, Yuri Takahashi ${ }^{2,3}$ and M Andrew Walker ${ }^{2}$
}

\author{
Address: ${ }^{1}$ USDA-ARS, 9611 S. Riverbend Avenue, Parlier, California 93648, USA, 2 Department of Viticulture \& Enology, University of California, \\ Davis, California 95616, USA and ${ }^{3}$ Department of Food sciences, Ehime Women's College, Uwajima 798-0025 Japan \\ Email: Hong Lin* - hlin@fresno.ars.usda.gov; Harshavardhan Doddapaneni - harsha@fresno.ars.usda.gov; \\ Yuri Takahashi - takahashi@aitan.ac.jp; M Andrew Walker - walker@ucdavis.edu \\ * Corresponding author †Equal contributors
}

Published: 22 February 2007

BMC Plant Biology 2007, 7:8 doi:10.1 186/147|-2229-7-8
Received: 23 August 2006

Accepted: 22 February 2007

This article is available from: http://www.biomedcentral.com/I47/-2229/7/8

(C) 2007 Lin et al; licensee BioMed Central Ltd.

This is an Open Access article distributed under the terms of the Creative Commons Attribution License (http://creativecommons.org/licenses/by/2.0), which permits unrestricted use, distribution, and reproduction in any medium, provided the original work is properly cited.

\begin{abstract}
Background: The gram-negative bacterium Xylella fastidiosa ( $\mathrm{Xf}$ ) is the causal agent of Pierce's disease (PD) in grape as well as diseases of many fruit and ornamental plants. The current molecular breeding efforts have identified genetic basis of PD resistance in grapes. However, the transcriptome level characterization of the host response to this pathogen is lacking.

Results: Twelve tissue specific subtractive suppression hybridization (SSH) cDNA libraries derived from a time course sampling scheme were constructed from stems, leaves and shoots of PD resistant and susceptible sibling genotypes $(V$. rupestris $\times V$. arizonica) in response to $X f$ infection. $A$ total of 5,794 sequences were obtained from these cDNA libraries from which 993 contigs and 949 singletons were derived. Using Gene Ontology (GO) hierarchy, the non-redundant sequences were classified into the three principal categories: molecular function (30\%), cellular components (9\%) and biological processes (7\%). Comparative analysis found variations in EST expression pattern between infected and non-infected PD resistant and PD susceptible grape genotypes. Among the three tissues, libraries from stem tissues showed significant differences in transcript quality suggesting their important role in grape-Xylella interaction.
\end{abstract}

Conclusion: This study constitutes the first attempt to characterize the Vitis differential transcriptome associated with host-pathogen interactions from different explants and genotypes. All the generated ESTs have been submitted to GenBank and are also available through our website for further functional studies.

\section{Background}

Pierce's disease (PD) has been a chronic problem for California's grape industry since the 1880s. The threat from this disease has recently become more severe with the introduction and establishment of a more effective vector, the glassy-winged sharpshooter (Homalodisca coagulate).
The disease is caused by Xylella fastidiosa, a xylem-limited, gram negative bacterium that is hosted by a wide range of plant species in and around vineyards in the southern United States and Mexico [1]. Over the past few years, federal, state governments, and the grape industry have funded PD research. Much of this research has focused on 
means of controlling the vector with insecticides and natural predators as a critical first step in integrated crop management. However, even low populations of the glassy-winged sharpshooter can have severe impact on vineyard health, thus limiting the effectiveness of predators to solve PD. In addition, as pesticide use becomes more restricted and as pesticide resistance develops, it is likely that the ultimate solution to PD will be host resistance.

Resistance to PD exists in some grape species and cultivars have been bred from these species. For example, accessions of Vitis aestivalis, V. arizonica, V. shuttleworthii, and $V$. smalliana are highly resistant to PD [2], and breeding programs have utilized these resistant species to develop PD resistant grapes for the southeastern United States [3]. Efforts to breed PD resistant grapes for California are underway [4]. The goals of these breeding efforts are to develop durably resistant cultivars, map and identify DNA-based markers for resistance to aid in selection, and to identify resistance genes. The introduction of PD resistance genes into wine grapes is complicated by the need for several generations of back-crossing to exclude unfavorable fruit characters associated with the resistant Vitis species. Once resistance genes are identified it may be possible to directly introduce resistance into elite wine grape cultivars by transgenic technologies.

Systemic infection studies under greenhouse conditions have shown differential distribution patterns of $X$. fastidiosa populations between resistant and susceptible genotypes and also among different organs or tissues of resistant genotypes [2]. This study found that $X$. fastidiosa populations in the tissues of susceptible genotypes did not differ among nodes, internodes, petioles, and leaf blades. However, the resistant genotypes had lower $X$. fastidiosa population levels, with highest levels in leaf blades, followed by petioles, and lowest levels in stem nodes and internodes. Differences between $X$. fastidiosa populations in the resistant genotypes compared to the susceptible genotypes were greatest in the stem internodes. The inheritance of PD resistance in a $V$. rupestris $\times V$. arizonica population was also evaluated by quantifying $X$. fastidiosa levels with ELISA [5] and by symptomology, including leaf scorch and a cane maturation index [2]. From genotypic screening and genetic mapping studies, it was concluded that a dominant allele controls PD resistance [5]. More recently, Krivanek et al. [6] have identified a locus that is linked to PD resistance and denoted it as 'Pierce's disease resistance 1' (PdR1). These studies confirm that there is genetically based PD resistance in grapes. They also found a range of resistance and tolerance to $X$. fastidiosa, which suggests that host responses to the pathogen are genotype dependent. The results from these studies prompted investigations into molecular basis of these host-pathogen interactions, which are currently poorly understood.

Functional genomic approaches provide powerful tools for identifying expressed genes. Among these techniques, expressed sequence tags (EST), [7], serial analysis of gene expression (SAGE), [8] and massively parallel signature sequencing (MPSS), [9], have been successfully employed. However due to its relative simplicity and ease, single pass EST sequencing has been the most widely used method to characterize genes associated with cellular development, biotic and abiotic stress in plant research.

Subtractive suppression hybridization (SSH) EST cloning can be used to maximize the identification of genes involved in host responses to pathogen infection and disease development. SSH cloning is also an effective method for cloning differentially regulated genes in cells. This technique has been used to isolate plant genes that are expressed in response to infection [10-12]. Using molecular hybridization and subtraction techniques, the SSH cDNA library approach reduces the cloning of abundantly expressed housekeeping genes or genes commonly expressed in both control and treated plants, thereby normalizing expressed cDNA profiles during library construction. As a result, it significantly enhances the chances of cloning differentially expressed genes. This is particularly important because many pathogenesis-related genes are expressed at low levels, and can be limited to a particular tissue or cell type [13]. These genes are less likely to be represented in a library if standard EST cloning methods are used. Recently completed EST projects have greatly contributed to the total number of developmentally regulated Vitis ESTs available in the public domain [14-16]. Further, there is information on microarray gene expression associated with viral infection [17] and on individual ESTs involved in host defense such as nonspecific lipid-transfer proteins (nsLTPs) [18] and phytoalexin [19]. However, to date information on ESTs expressed in response to the $X$. fastidiosa challenge is lacking.

The goal of this study was to characterize the molecular events in the grape/X. fastidiosa interaction using the SSH technique to compare populations of mRNA from highly resistant and susceptible grape genotypes from a grape mapping population being used to characterize PD resistance derived from a $V$. arizonica $\times V$. candicans hybrid [5]. For instance, the identified putative genes that are associated with host defense and/or resistance responses in this study can be used to develop molecular markers for PD resistance genetic mapping project. They are also useful for molecular-assistant-selection if they are found to be tightly linked to the PD resistance genes. To maximize cloning expression profiles associated with the host-pathogen interaction, a time course sampling scheme was 
designed tissue specific cDNA libraries were constructed from stem, leaf and shoot tissues. This report provides the transcriptome analysis of contrasting genotypes in response to $X$. fastidiosa infection among different tissues and provides ESTs associated with this host-pathogen interaction.

\section{Results}

\section{Sequencing and assembly}

A total of 5,794 ESTs with an average of 482 ESTs per library were sequenced from the $12 \mathrm{SSH}$ libraries. The average size of the EST was 282 bp with 5,421 sequences of $100 \mathrm{bp}$ or more. The number of ESTs sequenced from each library varied from 290 to 715 sequences (Table 1). Transcript redundancy in the EST collection was reduced by first comparing clusters within each library and then among all 12 libraries. These comparisons resulted in the assembling of 1,942 unique sequences including 993 clusters (contigs) and 949 singleton ESTs (Table 1). The percentage of unique sequences in each library varied from 19.3 to $74.5 \%$ (Table 1). In the resistant genotype 9621-67, transcript diversity from leaf and shoot tissues was reduced from 74.5 to $28.96 \%$ and from 37.96 to $21.4 \%$, respectively, after infection by $X$. fastidiosa. However the opposite results were observed in the stem tissue where transcript diversity increased from 43.3 to $57.7 \%$ (Table 1). In the susceptible genotype, on the other hand, transcript diversity was reduced in infected leaf and stem tissues and also in the non-infected shoot tissue (Table 1).

In order to assess the number of unique and overlapping transcripts among the 12 libraries, four comparisons were made: those derived from resistant infected (RI)-libraries (libraries, 1, 2 and 9); those derived from resistant control (RC)-libraries (libraries, 4, 5 and 11); those derived from susceptible infected (SI)-libraries (libraries, 3, 7 and 12); and those from susceptible control (SC)-libraries (libraries, 6, 8 and 10).
There were a total of 1561 contigs 338, 440, 336 and 447 that were further assembled into the four respective classes 305 (RI), 389 (RC), 294 (SI) and 413 (SC). These sequences were later used to construct the 993 non-redundant contigs for all 12 libraries (Table 1). Singletons were not included in this analysis. Contigs were grouped as present in one, two, three or all the four classes (Figure 1).

The number of non-overlapping sequences in the above four classes was 141 (RI), 212 (RC), 135 (SI) and 225 (SC), respectively. Only 31 sequences were common among all four classes; 39 contigs had ESTs that were expressed in the two control classes (RC and SC) and 22 had ESTs common between the two infected classes (RI and SI) (Figure 1). The distribution also included 32 contigs that were made from SI and RC classes and 37 contigs that were made from RI and SC classes. After this analysis, $72 \%$ of the 993 unique contigs belonged to one of the above four class, while the remaining $28 \%$ were overlaps.

\section{Functional annotation of the ESTs and comparative expression analysis}

Comparison of the 1,942 non-redundant sequences from the SSH libraries against the non-redundant protein database (nr) of the NCBI revealed that 716 sequences have significant similarity $\left(\leq 1 \mathrm{E}^{-5}\right)$ to existing sequences and 1,226 were unique. Only two ESTs showed significant similarity to $X$. fastidiosa (Additional file 1). Complete details of the blast results are available through our website [20].

When these 1,942 sequences were passed through the HtGo-Fat toolkit and BLAST searched against the supplied database, 915 sequences generated a hit, out of which 904 had at least one GO term (Additional file 2). Based on the generated GO information, these 904 sequences were divided in to the three principal GO categories: molecular function $(30 \%)$, cellular component $(9 \%)$ and biological

Table I: Summary of the ESTs generated from the 12 grape SSH libraries.

\begin{tabular}{|c|c|c|c|c|c|c|c|c|}
\hline Group Category & Lib I.D. & Library description & Total ESTs sequenced & Contigs & Singletons & Non-redundant ESTs & Redundant (\%) & Unique (\%) \\
\hline \multirow[t]{3}{*}{$\mathbf{R I}$} & I & infected leaf $R$ & 487 & 89 & 15 & 104 & 78.64 & 21.36 \\
\hline & 2 & infected stem $R$ & 504 & 177 & 114 & 291 & 42.26 & 57.74 \\
\hline & 9 & Infected shoot R & 404 & 72 & 45 & 117 & 71.04 & 28.96 \\
\hline \multirow[t]{3}{*}{$\mathbf{R C}$} & 4 & non-infected leaf $R$ & 324 & 95 & 28 & 123 & 62.04 & 37.96 \\
\hline & 5 & non-infected stem $\mathbf{R}$ & 586 & 175 & 79 & 254 & 56.66 & 43.34 \\
\hline & 11 & non-infected shoot $R$ & 415 & 170 & 139 & 309 & 25.54 & 74.46 \\
\hline \multirow[t]{3}{*}{ SI } & 7 & infected leaf S & 611 & 86 & 32 & 118 & 80.69 & 19.31 \\
\hline & 3 & Infected stem S & 290 & 90 & 23 & 113 & 61.03 & 38.97 \\
\hline & 12 & Infected shoot S & 446 & 160 & 136 & 296 & 33.63 & 66.37 \\
\hline \multirow[t]{5}{*}{ SC } & 8 & non-infected leaf S & 589 & 155 & 160 & 315 & 46.52 & 53.48 \\
\hline & 6 & non-infected stem $\mathbf{S}$ & 715 & 233 & 150 & 383 & 46.43 & 53.57 \\
\hline & 10 & non-infected shoot $S$ & 423 & 59 & 28 & 87 & 79.43 & 20.57 \\
\hline & Total & & 5794 & $|56|$ & 949 & & & \\
\hline & Unique & & & 993 & & & & \\
\hline
\end{tabular}

The percent unique and redundant ESTs was calculated for each library. Resistant and susceptible genotypes are tagged with "R" and "S" for library description. 


\section{RC- 389}

\section{RI- 305}

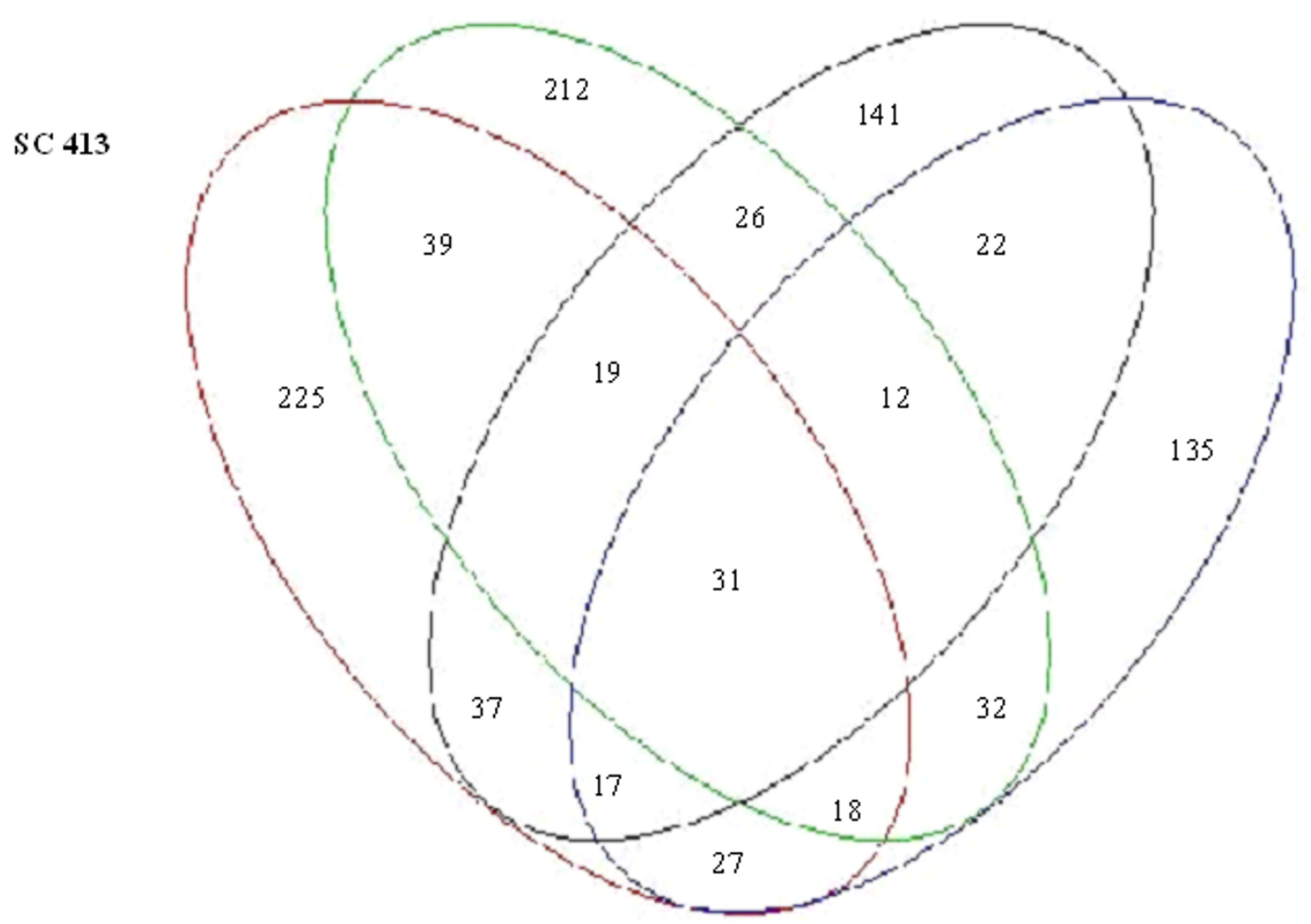

SI - 294

\section{Figure I}

Co-expression pattern of the ESTs. The 12 SSH libraries were grouped into four classes: resistant infected (RI)-libraries I, 2 and 9; resistant control (RC)-libraries 4, 5 and II; susceptible infected (SI)- 3, 7 and I2; and susceptible control (SC)- 6, 8 and 10 libraries. The distribution of the ESTs that were used to generate the 993 non-redundant contigs was plotted among the four classes.

process $(7 \%)$ (Figure 2A). Under the molecular function category, ligand binding and carrier protein contributed for $27 \%$ of the total contigs followed by the ribosomal coding transcripts 15\% (Figure 2B). Transport sequences $24 \%$ followed by signal transduction and defense response sequences $19 \%$ accounted for the majority of those in the biological process category, while many of the sequences in the cellular component category were in the chloroplast 30\%, membrane and nucleus subsections $26 \%$ (Figure 2C\&2D). More than half of the sequences (54\%) did not match sequences in the existing databases (Fig 2A) and other sequences were divided among the three principle categories. The full list of gene annotation along with the corresponding GO terms can be queried through our website [20].

Non-redundant sequences (contigs and singleton ESTs) from each individual library were analyzed using the GO classification. In order to address the issue of uneven EST numbers from each library, we compared relative abundance of the gene function categories based on their relative proportions from different library types (Table 2). Among the leaf tissue libraries, the non-infected leaf RC library was significantly different from the other leaf libraries because of the higher percentage of ESTs representing signal transduction and defense response (6.5), xenobiotic metabolism (3.25), nutrient reservoir activity (3.25), hydrolase activity and hydrolyzing O-glycosyl compounds (2.44). Infected leaf (RI) libraries showed multi-fold over expression of the monoxygenase/oxydoreductase activity $(10.17-10.58)$ related ESTs compared to the non-infected leaf libraries (Table 2).

Comparison of the four stem libraries showed that the SI library differed significantly for ESTs related to xenobiotic metabolism (13.27), nutrient reservoir activity (7.08) and 


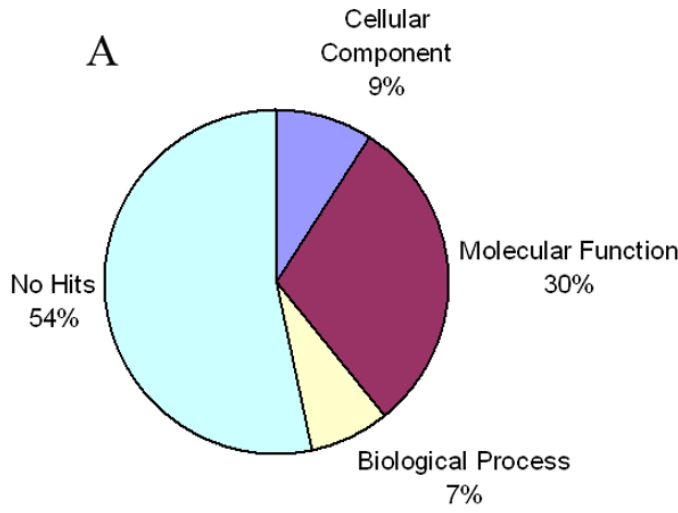

B

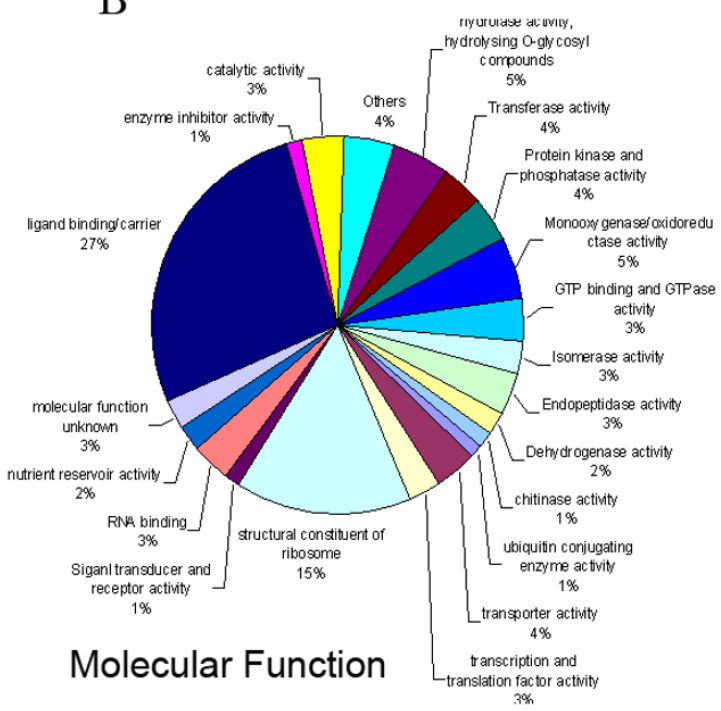

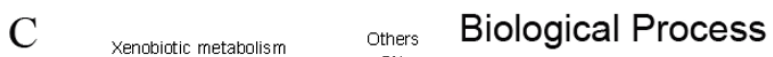

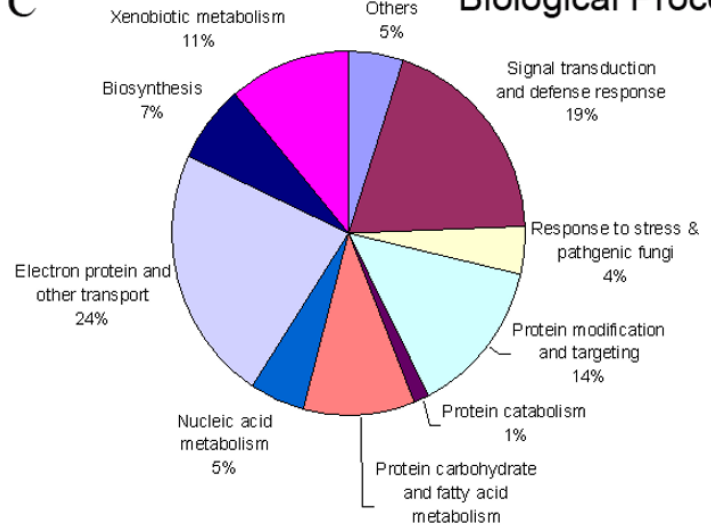

D $10 \%$

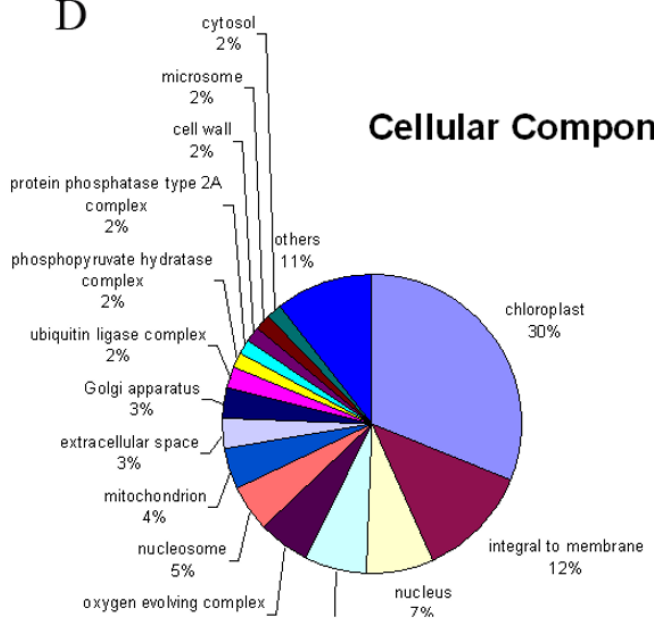

\section{Figure 2}

Percentage representation of gene ontology (GO) mappings for the 962 I-67 and 962 I-94 hybrids clusters. Functional annotation was carried using the High Throughput Gene Ontology Functional Annotation (Ht-Go-Fat) toolkit. The pie diagrams show the distribution of 905 sequences among the three principal GO categories. EST distribution (A) among the three GO principles (B) Molecular Function (C) Biological Process (D) Cellular Component.

monooxygenase/oxidoreductase activity (3.54). In contrast, ligand binding/carrier EST category was markedly lower than for that of the other three libraries. Control libraries from both the genotypes had a higher percentage of the transport related ESTs (3.13-3.54) compared to the infected libraries (Table 2).

Among the four shoot libraries, the RI was significantly different for ESTs of protein modification and targeting (2.56) and monooxygenase/oxidoreductase activity (6.84) in comparison to the other three libraries while the SI shoot library differed for protein kinase and phos- phatase activity ESTs compared to the other three libraries.

Interestingly, stem libraries showed a higher percentage of the signal transduction and defense-related response ESTs than the leaf and shoot libraries (Table 2). With the exception of the RC leaf library, chloroplast related ESTs were abundant in the leaf libraries.

In order to evaluate the diversity and specificity of the transcripts that were specific to a physiological condition, individual library specific ESTs were studied. There were 
Table 2: Distribution of differentially expressed ESTs among the three tissue types. Non-redundant sequences (contigs and singleton ESTs) from each individual library were analyzed using the GO classification.

\begin{tabular}{|c|c|c|c|c|c|c|c|c|c|c|c|c|}
\hline \multirow[t]{2}{*}{ Biological process } & \multicolumn{4}{|c|}{ leaf } & \multicolumn{4}{|c|}{ stem } & \multicolumn{4}{|c|}{ shoot } \\
\hline & lib-I & lib-4 & lib-7 & lib-8 & lib-2 & lib-5 & lib-3 & lib-6 & lib-9 & lib-I I & lib-I 2 & lib- 10 \\
\hline & Inf-Res & Cont-Res & Inf-Sus & Cont-Sus & Inf-Res & Cont-Res & Inf-Sus & Cont-Sus & Inf-Res & Cont-Res & Inf-Sus & Cont-Sus \\
\hline $\begin{array}{l}\text { Signal transduction and defense } \\
\text { response }\end{array}$ & & 6.50 & & 0.32 & 3.09 & 3.15 & 5.31 & 2.35 & & & 0.68 & \\
\hline Response to stress \& pathgenic fungi & & & 0.85 & & 0.34 & 0.39 & & 0.52 & & 0.97 & 0.34 & \\
\hline Protein modification and targeting & 1.92 & & 2.54 & & 1.37 & 1.97 & & 0.78 & 2.56 & 0.97 & 0.68 & \\
\hline Protein catabolism & & & & & & & & & & 0.32 & 0.34 & \\
\hline $\begin{array}{l}\text { Protein carbohydrate and fatty acid } \\
\text { metabolism }\end{array}$ & 0.96 & 0.81 & & 0.63 & 1.03 & 0.39 & & 1.04 & & 0.65 & 0.68 & \\
\hline Nucleic acid metabolism & & 0.81 & & & 0.34 & 0.79 & & 0.52 & & 0.32 & 0.68 & \\
\hline Electron protein and other transport & 0.96 & 0.81 & 0.85 & 1.27 & 0.69 & 3.54 & 0.88 & 3.13 & & 1.62 & 1.69 & 2.30 \\
\hline Biosynthesis & & & 1.69 & 0.32 & & 0.39 & & 0.52 & & 0.32 & 1.02 & \\
\hline Xenobiotic metabolism & & 3.25 & & & 0.34 & 0.39 & 13.27 & 0.26 & & 0.32 & & 1.15 \\
\hline Others & & & & 0.32 & 0.34 & 1.57 & & & & 0.32 & 0.68 & \\
\hline \multirow[t]{2}{*}{ Cellular component } & \multicolumn{4}{|c|}{ leaf } & \multicolumn{4}{|c|}{ stem } & \multicolumn{4}{|c|}{ shoot } \\
\hline & lib-I & lib-4 & lib-7 & lib-8 & lib-2 & lib-5 & lib-3 & lib-6 & lib-9 & lib-I I & lib- 12 & lib- 10 \\
\hline Chloroplast & 11.54 & & 15.25 & I I.75 & 0.34 & & 0.88 & 0.78 & 3.42 & 2.59 & 4.41 & 8.05 \\
\hline Integral to membrane & & 0.81 & & & 1.37 & 4.72 & 0.88 & 1.04 & & 0.65 & 0.68 & \\
\hline Nucleus & 2.88 & & 0.85 & & 1.37 & 0.39 & & 0.78 & 0.85 & 0.32 & 0.34 & 1.15 \\
\hline Membrane & 1.92 & & & & 0.69 & 1.57 & & 0.52 & & & 1.69 & \\
\hline Oxygen evolving complex & 2.88 & & 1.69 & 1.59 & & & & 0.26 & & 0.32 & 0.34 & 1.15 \\
\hline Nucleosome & 2.88 & & 1.69 & 0.32 & 0.69 & & & 0.52 & & & & \\
\hline Mitochondrion & 0.96 & & & & 0.69 & 0.39 & & 0.52 & & 0.32 & 1.02 & \\
\hline Extracellular space & & & 0.85 & 0.32 & 1.03 & 0.39 & & 0.78 & & 0.32 & 0.34 & 1.15 \\
\hline Golgi apparatus & & & & & 0.34 & 0.39 & & 0.26 & & 0.65 & 0.68 & \\
\hline Ubiquitin ligase complex & & & & 0.63 & & 0.39 & & 0.26 & & & & \\
\hline Phosphopyruvate hydratase complex & & & & & & 0.39 & & 0.26 & & & 0.34 & \\
\hline \multirow[t]{3}{*}{ Protein phosphatase type $2 \mathrm{~A}$ complex } & & & & & & 0.39 & & 0.00 & & 0.65 & & \\
\hline & \multicolumn{4}{|c|}{ leaf } & \multicolumn{4}{|c|}{ stem } & \multicolumn{4}{|c|}{ shoot } \\
\hline & lib- I & lib-4 & lib-7 & lib-8 & lib-2 & lib-5 & lib-3 & lib-6 & lib-9 & lib-I I & lib- 12 & lib- 10 \\
\hline Cell wall & & & & & & & & 0.52 & 1.71 & & 0.34 & 1.15 \\
\hline Microsome & & & & & & & & 0.52 & & & 0.34 & \\
\hline Cytosol & & & & & 0.34 & & & 0.26 & & & & 1.15 \\
\hline Chromatin & & & 0.85 & & & & & & & & 0.34 & \\
\hline Extracellular region & & 1.63 & & & & 0.39 & & & & & & \\
\hline Mitochondrial inner membrane & 0.96 & & & & & & & & & & 0.34 & \\
\hline Plasma membrane & & & & 0.32 & & & & & 0.85 & & & \\
\hline Photosystem I & & & & 0.63 & & & & & & & & \\
\hline Photosystem I reaction center & & & 0.85 & 0.63 & & & & & & & & \\
\hline $\begin{array}{l}\text { Ribulose bisphosphate carboxylase } \\
\text { complex }\end{array}$ & & 0.81 & & & & 0.39 & & 0.26 & & & & \\
\hline
\end{tabular}


Table 2: Distribution of differentially expressed ESTs among the three tissue types. Non-redundant sequences (contigs and singleton ESTs) from each individual library were analyzed using the GO classification. (Continued)

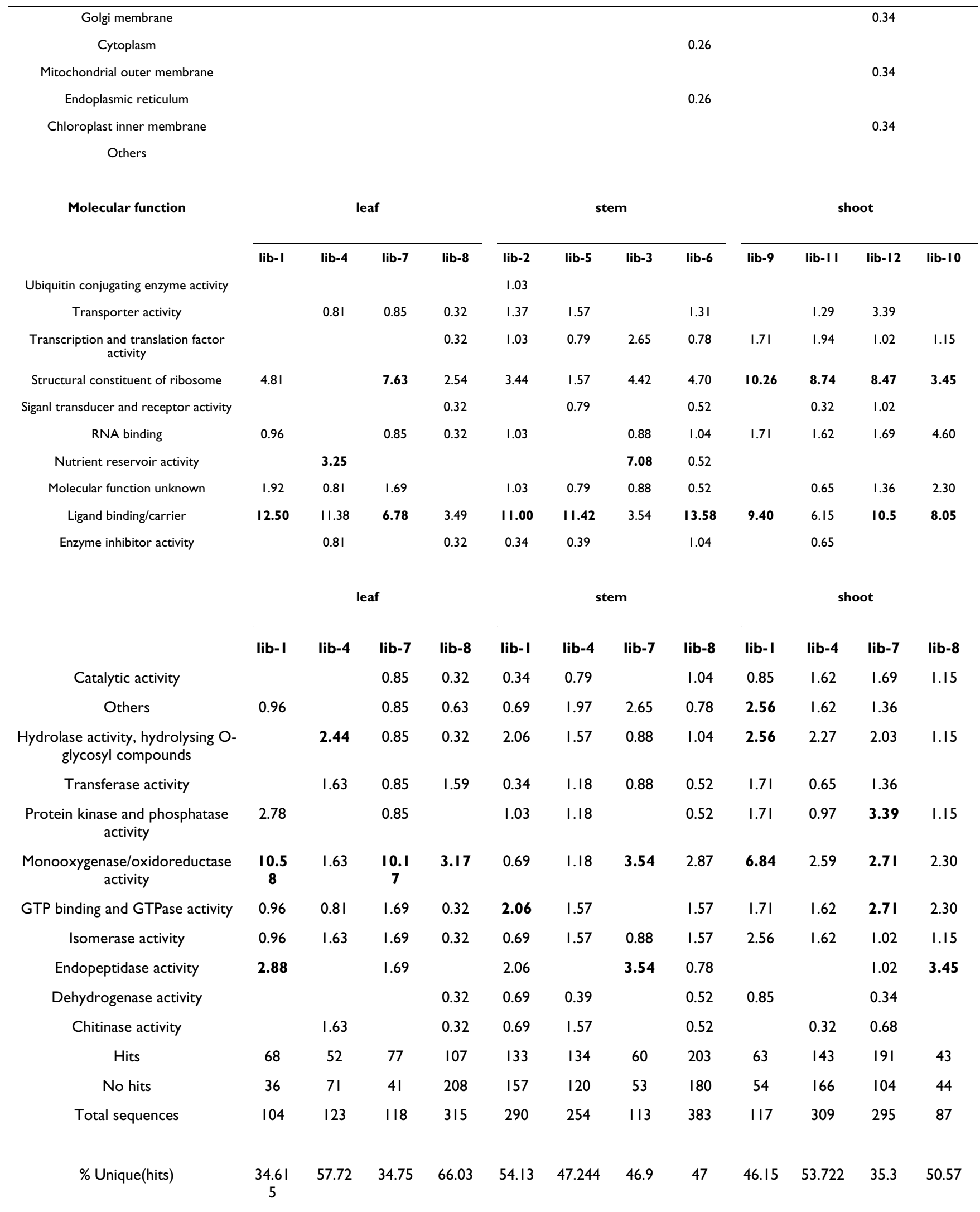


Table 3: Real-time quantitative RT-PCR results of the eight randomly selected ESTs from the SSH libraries.

\begin{tabular}{|c|c|c|c|c|c|c|c|c|}
\hline \multirow[t]{2}{*}{ Days (post inoculation) } & \multicolumn{8}{|c|}{ Fold Change (infected/control) } \\
\hline & Contig I 01 & Contig852 & Contig750 & LII_67_Sh_CT & Contig748 & Contig732 & Contig7 I0 & Contig935 \\
\hline 94-leaf-I-day & $2.2 \pm 1.5$ & 1.0 & $-2.7 \pm 0.0$ & 1.0 & 1.0 & 1.0 & 1.0 & 1.0 \\
\hline 94-leaf-3-weeks & $3.7 \pm 1.7$ & $4.2 \pm 1.0$ & 1.0 & 1.0 & $2.4 \pm 2.3$ & 1.0 & 1.0 & $-2.7 \pm 1.3$ \\
\hline 94-leaf-8-weeks & $13.6 \pm 1.0$ & $10.1 \pm 1.0$ & $17.0 \pm 1.2$ & 1.0 & $15.9 \pm 1.4$ & $2.1 \pm 1.7$ & 1.0 & 1.0 \\
\hline 67-leaf-I-day & $-2.4 \pm 0.8$ & - & 1.0 & 1.0 & $-2.3 \pm 0.8$ & $3.8 \pm 1.4$ & 1.0 & 1.0 \\
\hline 67-leaf-3-weeks & 1.0 & $2.2 \pm 1.2$ & 1.0 & 1.0 & 1.0 & 1.0 & 1.0 & $-12.6 \pm 1.1$ \\
\hline 67-leaf-8-weeks & 1.0 & - & $-2.5 \pm 1.5$ & 1.0 & 1.0 & 1.0 & 1.0 & 1.0 \\
\hline 94-stem-I-day & 1.0 & $4.2 \pm 6.2$ & 1.0 & 1.0 & 1.0 & - & 1.0 & $6.6 \pm 1.0$ \\
\hline 94-stem-3-weeks & $30.3 \pm 2.7$ & $25.6 \pm 1.2$ & $23.5 \pm 2.3$ & $2.0 \pm 0.7$ & $5.2 \pm 1.7$ & $-118.6 \pm 2.9$ & 1.0 & $-56.5 \pm 1.2$ \\
\hline 94-stem-8-weeks & $464.7 \pm 1.0$ & $192.7 \pm 1.1$ & $94.0 \pm 1.2$ & 1.0 & $262.3 \pm 1.2$ & 1.0 & 1.0 & 1.0 \\
\hline 67-stem-I-day & $-2.4 \pm 0.5$ & $-2.7 \pm 0.3$ & 1.0 & $-4.5 \pm 2.2$ & $-2.0 \pm 1.8$ & $-6.6 \pm 1.2$ & 1.0 & 1.0 \\
\hline 67-stem-3-weeks & $2.5 \pm 1.6$ & $3.7 \pm 1.3$ & $34.2 \pm 1.0$ & 1.0 & $11.2 \pm 1.8$ & $-3.2 \pm 3.5$ & 1.0 & 1.0 \\
\hline 67-stem-8-weeks & $-4.4 \pm 1.7$ & 1.0 & $11.9 \pm 3.3$ & 1.0 & $3.0 \pm 1.6$ & $-8.6 \pm 2.2$ & 1.0 & $-2.9 \pm 1.34$ \\
\hline
\end{tabular}

RNA from two tissues (stem and leaf) at three stages of development (I day, 3 weeks and 8 weeks post infection) from both resistant (67) and susceptible (94) genotypes were analyzed. Results presented here are the mean \pm SD values of biological replicates. Fold differences were calculated for $\mathrm{Ct}$ values of infected over control RNA samples. Values for less than two-fold change were entered as (I.0). For annotation and primer sequence details, please refer to Table-4.

949 singleton ESTs and 689 contigs that fell into this category. The stem libraries showed reduced transcript diversity following $X$. fastidios $a$ infection in both the resistant (Lib-5 and Lib-2) and susceptible selections (Lib-3 and Lib-6). While the control libraries had a wide range of functional ESTs including pathogen related (PR) proteins in both the selections, infected libraries were enriched with PR proteins. The resistant infected libraries also were more diverse than the susceptible infected libraries. The resistant stem infected library had transcripts encoding PR protein such as $\beta 1-3$ glucanase and $14 \mathrm{kDa}$ proline-rich protein, primary cell wall modifying proteins such as, xyloglucan endotransglycosylase (XET), endoxyloglucan transferase (EXT), and metabolic enzymes such as cinnamoyl-CoA reductase, isopropylmalate dehydrogenase, glutamate decarboxylase, 3-hydroxybutyryl-CoA dehydrogenase, PEP carboxylase, quinine reductase, and auxin responsive factor that appeared following $X$. fastidiosa infection. On the other hand, the susceptible infected stem library was over represented by transcripts encoding PR proteins such as PR-23S NP24 protein precursor and osmotin-like protein TPM-1, glucan 1,3-beta-glucosidase, seed storage legumin like protein and proteolytic pathway proteins such as aspartic protease, beta7 proteasome subunit and 20S proteasome beta subunit (PBG1) that were absent in the control library. The infected leaf libraries were free of any known transcripts of PR proteins, with control libraries having a greater percentage of transcripts encoding unknown proteins compared to the infected libraries. Only the SI shoot library had pathogen responsive ESTs (a chitinase-like protein, a nonspecific lipidtransfer protein precursor (LTP) and an F-box/LRR-repeat protein-20). The RI shoot library did not have any of the above transcripts in this given transcriptome set.

\section{Real-Time Quantitative RT-PCR analysis of the differential expression}

RT-PCR analysis of 7 out of the 8 selected ESTs confirmed differential expression under the conditions studied. Four out of the eight ESTs had greater expression in the susceptible variety, with gradual accumulation of the transcript as the disease progressed (Table 3). Expression of these ESTs was much higher in the stem tissue than in the leaf tissue, particularly at 8-weeks post inoculation. Two of these ESTs were annotated as encoding PR proteins, while the other two appeared to be novel (Table 3). Three transcripts involved in the cell homeostasis, two belonging to the metallothionin family and a SOS2 protein kinase that is required for sodium and potassium ion homeostasis and salt tolerance in plants, showed a different trend. Expression of both the ESTs of metallothionin family was down regulated in the stem tissue at 8 weeks after inoculation in both the susceptible and resistant genotypes, while the response varied for other stages suggesting different functional roles for these two transcripts. In contrast, the expression of SOS2 protein kinase EST did not vary significantly in this process (showed less that 2-fold variation). Expression of the L11_67_Sh_CT was down regulated $(-4.48 \pm 2.02)$ in the resistant 9621-67 stem samples collected $24 \mathrm{~h}$ post inoculation. This EST had sequence similarity to mitogen-activated protein kinase kinase (MAPKK) that was cloned from the control RC shoot library of the same genotype.

\section{Discussions and Conclusion}

This study constitutes the first genome-wide effort to understand the molecular basis of a host- $X$. fastidiosa interaction in Vitis. Twelve forward and reverse suppression subtractive cDNA libraries from two genotypes 
(resistant and susceptible) for three different tissues and 10 different stages of Pierce's disease development were constructed to identify spatial and temporal transcriptional changes resulting from $X$. fastidiosa infection. Because a whole Vitis genome sequence is not yet been completed, ESTs could serve as an efficient alternative approach to the discovery of novel genomic information. Out of the 1,942 non-redundant ESTs that were cloned in this study, about 33\% were found to be unique, demonstrating the effectiveness of the experimental design and the construction strategy utilized for these SSH libraries. RT-PCR analysis of seven out of the eight selected ESTs from SSH confirmed their differential expression under the test conditions. Five out of the six transcripts showed up regulation in the tissue types and condition from which they were cloned. However, the number of transcripts that were cloned for each of these ESTs (based on the ESTs that were used in generating the contig) was several folds lower than their original numbers (as indicated by the RT-PCR change values) in the RNA pool, indicating that suppression of the EST numbers that appeared in the final pool was effective. Furthermore, more than half $(54 \%)$ of these sequences did not match the sequences in the GenBank and 508 were not reported in the Vitis EST database collection and are therefore unique contributions to the Vitis EST pool. A significant difference in the number and diversity of transcripts was observed in response to $X$. fastidiosa infection in the resistant vs. susceptible genotypes, suggesting host responses to infection are genotype dependent. The present study identified a group of transcripts that are regulated in response to $X$. fastidiosa infection and may represent the key elements in development of the defense response.

There was a significant reduction in transcript diversity, particularly in leaf tissues, in both the resistant and susceptible genotypes, after infection with $X$. fastidiosa (Table $1)$. This transcript variation was supported by the coexpression pattern of the ESTs with only $28 \%$ of the ESTs overlapping among the four classes and the rest being unique to each of those classes (Figure 1). The large percentage of transcripts involved in ligand binding, carrier signal transduction, and defense response among the annotated transcripts from the inoculated tissue also supports the presumption that many of these transcripts are specifically involved in the $X$. fastidiosa resistance response. These observations are consistent with previously reported studies on host-pathogen interactions $[21,22]$.

Among the three tissue types, comparisons between libraries from resistant and susceptible infected stem tissues produced the most interesting EST expression patterns. The resistant library had ESTs with primary cell wall modifying and metabolic enzymes and for known PR proteins such as $\beta$ 1-3 glucanase.

Plant cell elongation depends on physical properties of the primary cell wall. The class of enzymes, called alternatively endo-xyloglucan transglycosylase (EXT) or xyloglucan endotransglycosylase (XET), modifies xyloglucan (XG) by cleavage and rejoining of the $\beta(1-4)$-XG backbone. Such activity can potentially alter cell size by loosening or tightening of the cell wall. Enzymes with XET activity have been identified in rapidly growing tissues from various plant species [23] and multigene families related to XET have been identified [24,25]. Expression of primary cell wall modifying ESTs in the RI stem library, suggest active modification and expansion of cell wall tissues. Such cell wall modifications have been hypothesized to be physical barriers to limit further pathogen invasion [26]. Furthermore, expression of ESTs involved in cell metabolic activities might also reflect the pathogen's minor effect on tissue metabolism in these cells. The microarray comparative analysis study conducted by Bray [27] indicated that the xyloglucan endotransglucosylase/ hydrolases (XTHs) family of genes was down regulated under water deficit conditions in three independent experiments, supporting the non-water stressed nature of the RI plants.

Enhanced transcription of $\beta$ 1-3 glucanase activity in grape has been previously associated with exogenous application of ethephon, an ethylene precursor [28]. In a more recent study, Kortekamp [29] found that PR-2 ( $\beta$ 13 glucanase) expression was associated with responses to Pseudoperenospora cubensis infection in the resistant grape rootstock 'Gloire de Montpellier' (V. riparia) compared to the susceptible cultivar 'Riesling' ( $V$. vinifera). EST expression in the susceptible stem library involved expression of a different class of PR proteins (PR-23S NP24 protein precursor and osmotin-like protein TPM-1) and also had different levels of seed storage and proteolytic EST expression, compared to their control tissues. Seed storage proteins such as legumins and vicillins are synthesized and accumulated during seed maturation and due to their regulation by agents such as abscisic acid, are associated with developing desiccation tolerance that occurs during seed maturation [30]. Small protein ubiquitin (Ub) and the $26 \mathrm{~S}$ proteosome, a 2-MDa protease complex, are key components of the proteolytic pathway [31]. In response to pathogen attack, the $\mathrm{Ub} / 26 \mathrm{~S}$ proteosome pathway initiates programmed cell death to localize pathogen spread [31]. Activation of proteolysis pathway ESTs in response to the pathogen attack has been documented previously [32].

Some of the PR proteins such as chitinases and $14 \mathrm{kDa}$ proline-rich protein ESTs were cloned only from resistant 
stem libraries. While ESTs, such as PR-10, were cloned from infected and control stem libraries of both susceptible and resistant selections. Previous reports in grape on PR-10 (intracellular proteins with unknown enzymatic function) expression point to its constitutive pre-infection role in pathogen defense [29]. The previously described proline rich proteins or P-rich proteins in Arabidopsis [33] and in Drosophila [34] are known antimicrobial compounds. Further functional studies will be required to understand the specific role of these cloned PR proteins in resistant stem tissues during $X$. fastidiosa infection.

Krivanek and Walker [2] found that resistant stems host 60 -fold fewer $X$. fastidios a cells than susceptible stems. The EST profiles produced here found unhindered metabolic activity in the resistant stem tissues and the occurrence of seed storage and proteolytic pathway proteins in the susceptible stem tissues, both suggesting the existence of a response to infection. Although PR protein expression was observed in the susceptible tissues, the nature of this expression was different since few of the PR proteins expressed in the susceptible tissues overlapped with those from resistant tissues. This finding suggests that even susceptible genotypes have a systemic and broad host defense response mechanism that responds to $X$. fastidiosa infection, it does not prevent PD and must be augmented to achieve the resistance observed in 9621-67.

Four-way comparative analysis of the V. arizonica hybrid sequences with three other Vitis species contained in the GenBank EST collections (V. vinifera, $V$. shuttleworthii and $V$. aestivalis) revealed that $26 \%$ (508 ESTs) of the V. arizonica sequences were unique. There are 415 ESTs in common with $V$. vinifera (Unigene Built dated 04/13/06), 57 ESTs that were present in this set and the $V$. shuttleworthii set; and 24 ESTs that were also present in $V$. aestivalis set, but absent in the other two sets. In addition, there were 338 ESTs in common with the V. vinifera and $V$. shuttleworthii sets; 99 ESTs that were also present in $V$. vinifera and $V$. aestivalis sets, and 14 that were present also in $V$. shuttleworthii and $V$. aestivalis sets. The rest of the ESTs were found in all four sets.

This is the first study to display the extent of EST transcript diversity in grape after infection by $X$. fastidiosa. A fourway comparative analysis found that each of the EST collections had an independent niche with varying degrees of overlap with the set produced from $V$. arizonica. This study has identified likely molecular targets for developing PD resistant varieties and for characterizing their resistance genes. Based on the diversity and specificity of the presented EST cloning results, it is clear that stem tissue plays a prominent role in the $X$. fastidiosa grape interaction, supporting observations by Krivanek and Walker [2]. The generated ESTs with its unique collection will serve as an important addition to the grape transcript pool for further large scale expression studies.

\section{Methods}

\section{Plant materials and $X \boldsymbol{f}$ inoculation experiment}

Highly susceptible (9621-94) and resistant (9621-67) grape genotypes were selected from a mapping population segregating for resistance to $X$. fastidiosa. Resistance in this population derives from the $V$. rupestris $\times V$. arizonica/ $V$. candicans parent, F8909-17. This resistant selection [5] is a key parent in a PD resistance wine grape breeding program [4]. Herbaceous cuttings of both genotypes were rooted under mist-propagation and rooted plants were transplanted to 1 liter pots with a Yolo sandy loam/perlite/peat $(1: 1: 1)$ soil mix. Plants were maintained in a greenhouse at 24 to $32^{\circ} \mathrm{C}$ with 18 hours of exposure supplemented with High-Pressure Sodium lamp (20 watts per sq. ft.). Plants were watered twice daily with $160 \mathrm{ml}$ of water containing $25 \%$ Hoagland's solution (SigmaAldrich, St. Louis) using an automatic drip irrigation system. When plant shoots reached 30 to $40 \mathrm{~cm}$, they were pruned to two basal buds before regrowth to facilitate uniform plant growth.

A X. fastidiosa strain obtained from the Stag's Leap district of Napa Valley, California was used to inoculate the plants. The inoculation was carried out as described previously [2] with inoculum collected from five-day-old cultures growing on PW media [35] by washing with $\mathrm{ddH}_{2} \mathrm{O}$. Concentration of bacterial cells was adjusted to $6 \times 10^{8}$ $\mathrm{cfu} / \mathrm{ml}\left(\mathrm{A}_{600 \mathrm{~nm}}=0.25\right)$. Sixty plants from each treatment group were needle-inoculated with $10 \mu \mathrm{l}$ of bacterial suspensions in the stem at $10 \mathrm{~cm}$ above the base of plants. Sixty additional plants from each treatment group were inoculated with $\mathrm{ddH}_{2} \mathrm{O}$ from washed sterile PW plates.

\section{RNA isolation and SSH CDNA library construction}

Leaf, stem and shoot tip tissues were collected from five to six experimental plants at day 1,2 , and 5 post inoculation, and then at three 1 -week and four 2-week intervals. All the samples were immediately stored at $-80^{\circ} \mathrm{C}$ for later RNA extraction. PD symptoms began to develop on the susceptible genotype at about 6 weeks post inoculation and by 8 weeks, symptoms were severe. Total RNA was isolated from leaf, stem and shoot tissues using a modified CTAB extraction and lithium chloride precipitation as reported earlier $[36,37]$. The mRNA was isolated from total RNA using Dynabeads Oligo (dT) $)_{25}$ according to manufacturer's protocol (Dynal Biotech LLC., Brown Deer, WI USA). This step eliminated the possibility of DNA contamination in the RNA samples used for library construction. Purified mRNA samples were checked by gel and further evaluated with a BioAnalyzer. Only high quality mRNA was selected for cDNA synthesis. For each of the tissue, treatment and genotype sample, equal amounts of 


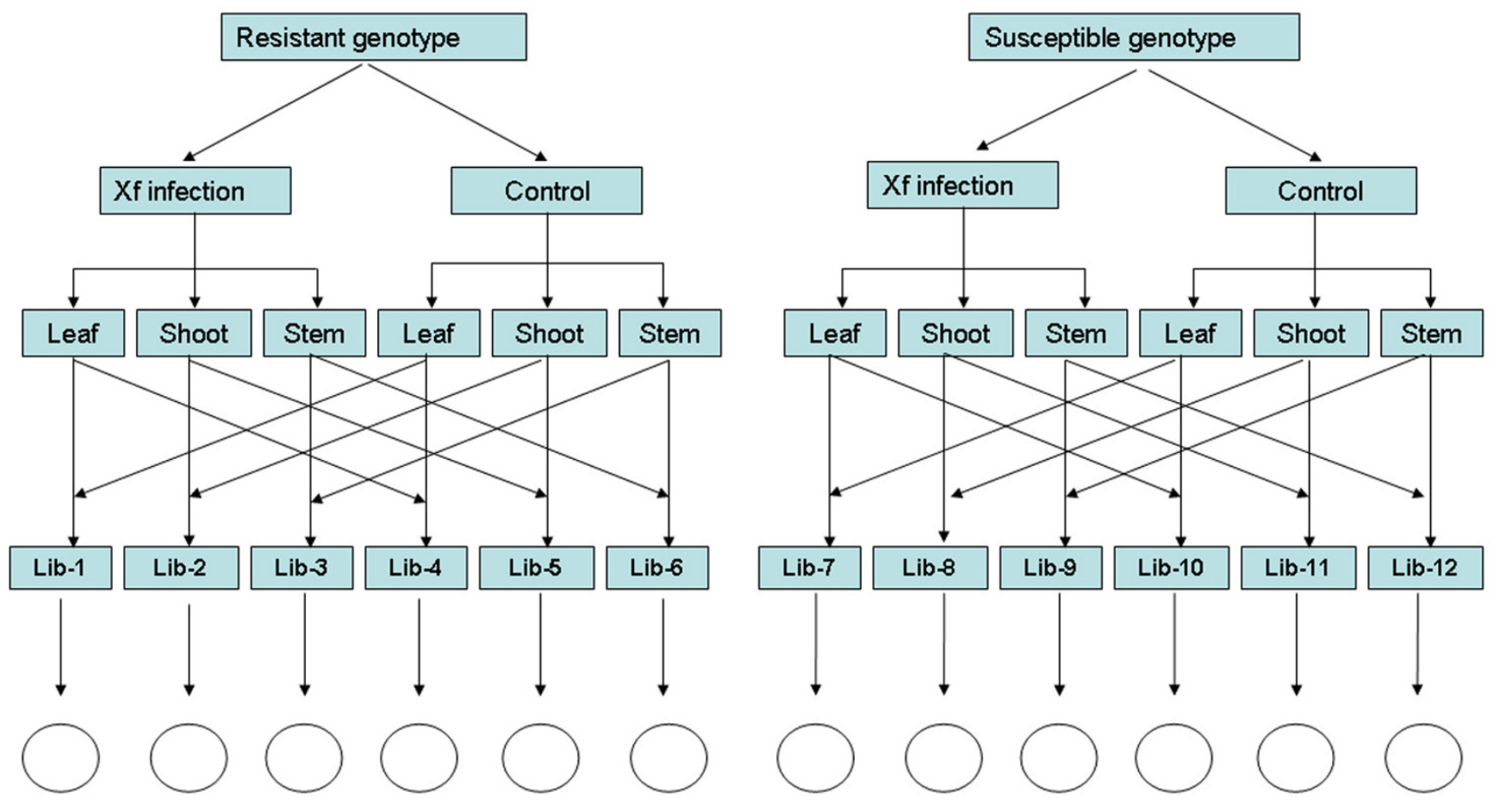

Figure 3

Strategy for SSH library construction. Twelve reciprocal tissue specific (stem, leaf and shoot) SSH cDNA libraries from highly resistant (962I-67) and highly susceptible (962I-94) sibling selections from a Vitis rupestris $\times$ V. arizonica population segregating for resistance to $X$. fastidiosa infection.

mRNA from time course experiments were pooled at this stage. Using 0.5 microgram of poly $(\mathrm{A})^{+}$from each sample and SMART ${ }^{\mathrm{mm}}$ CDNA synthesis kit (BD Bioscience, Palo Alto, CA), first and second strand cDNA followed by construction of forward and reverse subtractive suppression cDNA libraries was carried out per PCR-Select cDNA Subtraction Kit's (BD Bioscience, Palo Alto, CA) protocol. At the same time, suppression subtractive hybridization was optimized and used for each library construction.

\section{cDNA library sequencing, data analysis and dbEST submission}

To enhance the cloning of differentially expressed genes, 12 forward and reverse SSH cDNA libraries were constructed (Figure 3). Clones were randomly selected and single-pass sequenced with a vector primer upstream of the 5 '-end of the inserts. On an average, 500 clones were sequenced per library using ABI 3730 DNA Analyzer (Applied Biosystems, Foster City, CA, USA). Sequence chromatogram trace files were scored for quality with cutoff scores of Phred 20 using Base-Calling software. The FASTA files were trimmed of vector and adapter sequences. Non-target sequences such as rRNA and E. coli DNA were filtered out. The SSH cDNA libraries usually contained insertion sizes in a range of $200-700 \mathrm{bp}$. Qual- ity sequences greater than 100 bp were selected for further analysis. Sequence files were further analyzed to determine the number of contigs and EST singletons. After contig assembly, the dataset was searched using BLASTX program against the NCBI non-redundant protein database to search for coding protein homology. This analysis resulted in 5,421 sequences that were at least $100 \mathrm{bp}$ or more and had a Phred score of 20 or greater. These sequences were submitted to the GenBank EST database with the accession numbers [GenBank:DN942225 to DN947645].

ESTs were clustered and aligned into contigs and singlets using CAP3 initially from each library followed by comparison of the consensus contig sequences across all 12 libraries using MEGABLAST and grouped on the basis of similarity to clusters of related contigs. For further analysis, each cluster was represented by retaining all the consensus sequences of its contig members. Sequencing and part of contig generation were done at Macrogen Inc.

\section{EST Similarity search and functional assignments}

A similarity search against the NCBI 'nr' database was performed for each EST sequence using the BLASTX program with a cutoff $\mathrm{E}$ value of $10^{-4}$. Functional annotation was 
Table 4: Details of the primers used in this study.

\begin{tabular}{|c|c|c|c|c|}
\hline Sequence ID & Annotation & Sense Primer 5----3 & Anti-sense Primer 5---3 & $\begin{array}{l}\text { Amplicon } \\
\text { (bp) }\end{array}$ \\
\hline $\begin{array}{l}\text { HLCDTotal.seq.Contig } \\
\text { 101:60-443 }\end{array}$ & No hit & AAAGGGAAGTCCACACAGCC & CGAAACCACAAACTCCAAATCAAC & 199 \\
\hline $\begin{array}{l}\text { HLCDTotal.seq.Contig } \\
852: 60-447\end{array}$ & No hit & ACGCAAGAGGGAAAGAGAAG & GGGGAGCATAGTCTACTCCA & 92 \\
\hline $\begin{array}{l}\text { HLCDTotal.seq.Contig } \\
750: 60-307\end{array}$ & $\begin{array}{c}\text { Osmotin-like protein TPM-I precursor (PR } \\
\text { P23) (Fragment) }\end{array}$ & TAATAGAGGCTTTATGGGCAGAA & CTGATGCTTACAGCTACCCTAA & 100 \\
\hline $\begin{array}{l}\text { HLCDTotal.seq.Contig } \\
748: 60-265\end{array}$ & $\begin{array}{l}\text { NP24 protein precursor (Pathogenesis- } \\
\text { related protein PR P23) }\end{array}$ & GCAGAAGATAACATCATAGTTGGT & CCCTACAGAATACTCAAGGTATTT & 121 \\
\hline $\begin{array}{l}\text { LII_67_Sh_CT_PI_F0 } \\
8 . a b 1: 60-295 \text { I } 295\end{array}$ & $\begin{array}{c}\text { Mitogen-activated protein kinase kinase } \\
\text { MAPKK }\end{array}$ & ATTGTTTACAGATGAACGGATGCG & AGGGCGTATCGGAGGAGTTC & 111 \\
\hline $\begin{array}{l}\text { HLCDTotal.seq.Contig } \\
732: 60-421\end{array}$ & Metallothionein-like protein & TGCAGCCATTTCCGCATTTG & GCTCCTCAGAGAAAGCCACC & 144 \\
\hline $\begin{array}{l}\text { HLCDTotal.seq.Contig } \\
\text { 7|0:60-206 }\end{array}$ & SOS2-like protein kinase & AGGAAGATCTACAGAGGAGACTTT & TGGTTGGATCGAGGAGTTTCG & 88 \\
\hline $\begin{array}{l}\text { HLCDTotal.seq.Contig } \\
935: 60-374\end{array}$ & Putative metallothionein-like protein & GCTATGTTGGGACCGTTGTGA & GCCACAGGTGCAGTCAATACA & 98 \\
\hline
\end{tabular}

carried using the High Throughput Gene Ontology Functional Annotation (Ht-Go-Fat) toolkit [38]. This is an ontology based database built using functional classification schemes such as Gene Ontology (GO), Enzyme Commission numbers (EC), BioCarta Pathways, and Kyoto Encyclopedia of Genes and Genomes (KEGG) pathways was downloaded from the USDA-ARS website [38] and the sequence similarity search was carried out using the default blast parameters and a cut off $\mathrm{E}$ value of $10^{-4}$.

The Gene Ontology (GO) IDs for the sequences showing a hit were then separated from the blast file and the corresponding names and ontologies were extracted from the 'Gene Ontology.obo text file' downloaded from the GO web site [39]. Next, the GO categorization scheme of classification by biological process, cellular component and the molecular function was used to categorize the similarity results and to generate the representative pie diagrams. The full set of analysis files is made available through our VitisExpDB database [20]. VitisExpDB is an online MySQL-php driven relational database that houses annotated EST data for both $V$. vinifera and non-vinifera Vitis species. Using the latest Gene Ontology (GO) terminology, a uniform structural vocabulary was developed to cross reference several Vitis accessions. The database can be searched by Gene Ontology ID, GenBank ID, enzyme number, or by inputting keyword(s).

\section{Real-Time quantitative RT-PCR of the differentially expressed transcripts}

Gene transcripts for six of the randomly selected ESTs were quantified by real-time quantitative RT-PCR using the AB 7500 (Applied Biosystems, Foster City, CA, USA) and TaqMan One-Step RT-PCR Kit (Applied Biosystems, Foster City, CA, USA) per manufacturer's specifications. Total RNA (100 ng) extracted from leaf and stem tissues at three different stages of disease development ( 1 day, 3 weeks and 8 weeks post inoculation) from the susceptible
9621-94 and resistant 9621-67 was used for RT-PCR amplification using gene-specific primers (Table 4). Total RNA was normalized based on the ultra-sensitive RiboGreen $^{\circledast}$ RNA Quantitation Reagent (Molecular Probes, Inc. Eugene, USA) per the manufacture's protocol. This procedure was favored over gene expression based calibration because a spectrum of diseased stages vs healthy plant at different developmental stages was being compared. Furthermore, only values that were greater than 2-fold were considered significant. In the amplification reaction, SYBR Green 1 dye at $1 \times$ final concentration $\left(10^{-6} \mathrm{M}\right)$ was used for quantification. Reverse Transcription was carried out at $50^{\circ} \mathrm{C}$ for $30 \mathrm{~min}$. followed by PCR amplification for 35 cycles at $95^{\circ} \mathrm{C}$ for $15 \mathrm{sec}$ and annealing and extension at $60^{\circ} \mathrm{C}$ for $1 \mathrm{~min}$.

\section{Authors' contributions}

HL conceived of the study and along with YT carried out the greenhouse experiments, RNA preparation and construction of SSH libraries. HD did the data analysis, and RT-PCR experiments. AW and HL coordinated the project and along with HD wrote the manuscript. All the authors read and approved the final manuscript.

\section{Additional material}

\section{Additional file 1}

Summary of the BLAST search results for the non-redundant set of ESTs with NCBI 'nr' database. Summary table of the NCBI BLAST search. Click here for file

[http://www.biomedcentral.com/content/supplementary/14712229-7-8-S1.xls] 


\section{Additional file 2}

Summary of the GO BLAST search for the non-redundant EST set using HT-GO-FAT kit. Table with details of the GO annotation for the 915 ESTs. Sheet 2 lists the annotation details for all the 1940 sequences. Click here for file

[http://www.biomedcentral.com/content/supplementary/14712229-7-8-S2.xls]

\section{Acknowledgements}

We gratefully acknowledge the financial support from the California Department of Food and Agriculture's Pierce's Disease and Glassy-winged Sharpshooter Board. We appreciate the technical support provided by Rong $\mathrm{Hu}$, Samuel Barros and Parminder Sahota.

\section{References}

I. Purcell AH, Hopkins DL: Fastidious xylem-limited bacterial plant pathogens. Annu Rev Phytopathol 1996, 34:|3I-5I.

2. Krivanek AF, Walker MA: Vitis resistance to Pierce's disease is characterized by differential Xylella fastidiosa populations in stems and leaves. Phytopathology 2005, 95:44-52.

3. Mortensen JA: The inheritance of resistance to Pierce's disease in Vitis. J Am Soc Hortic Sci 1968, 92:331-337.

4. Walker MA, Tenscher A: Breeding Pierce's disease resistant winegrapes. Proceedings of the 2005 Pierce's Disease Research Symposium. San Diego, CA; 2005:77-80.

5. Krivanek AF, Famula TR, Tenscher A, Walker MA: Inheritance of resistance to Xylella fastidiosa within a Vitis rupestris $\times$ Vitis arizonica hybrid population. Theor Appl Genet 2005, I I I: I I 0-I I 9

6. Krivanek AF, Riaz S, Walker MA: Identification and molecular mapping of PdRI, a primary resistance gene to Pierce's disease in Vitis. Theor Appl Genet 2006, I | 2: | | 25- I I 3 |

7. Adams MD, Kelley JM, Gocayne JD, Dubnick M, Polymeropoulos MH, Xiao $\mathrm{H}$, Merril CR, Wu A, Olde B, Moreno RF, Kerlavage AR, McCombie WR, Venter JC: Links Complementary DNA sequencing: expressed sequence tags and human genome project. Science |99|, 252:165|-1656.

8. Velculescu VE, Zhang L, Vogelstein B, Kinzler KW: Serial analysis of gene expression. Science 1995, 270:484-487.

9. Brenner S, Johnson M, Bridgham J, Golda G, Lloyd DH, Johnson D, Luo S, McCurdy S, Foy M, Ewan M, Roth R, George D, Eletr S, Albrecht G, Vermaas E, Williams SR, Moon K, Burcham T, Pallas M, DuBridge RB, Kirchner J, Fearon K, Mao J-l, Corcoran K: Gene expression analysis by massively parallel signature sequencing (MPSS) on microbead arrays. Nat Biotechnol 2000 18:630-634.

10. Birch PRJ, Avrova AO, Lyon GD, Duncan JM, Toth RL: Isolation of potato genes that are induced during an early stage of the hypersensitive response to Phytophthora infestans. Mol PlantMicrobe Interact 1999, I 2:356-361.

II. Xiong L, Lee MW, Qi M, Yang Y: Identification of defense-related rice genes by suppression subtractive hybridization and differential screening. Mol Plant Microbe Interact 200I, 14:685-692.

12. Degenhardt J, Al-Masri AN, Kurkcuoglu S, Szankowski I, Gau AE: Characterization by suppression subtractive hybridization of transcripts that are differentially expressed in leaves of apple scab-resistant and susceptible cultivars of Malus domestica. Mol Genet Genomics 2005, 273:326-335.

13. Caturla M, Chaparro C, Schroeyers K, Holsters M: Suppression subtractive hybridization to enrich low-abundance and submergence-enhanced transcripts of adventitious root primordia of Sesbania rostrata. Plant Sci 2002, 162:915-921.

14. Moser C, Segala C, Fontana P, Salakhudtinov I, Gatto P, Pindo M, Zyprian E, Toepfer R, Grando MS, Velasco R: Comparative analysis of expressed sequence tags from different organs of Vitis vinifera L. Funct Integr Genomics 2005, 5:208-17.

15. Goes da Silva F, landolino A, Al-Kayal F, Bohlmann MC, Cushman MA, Lim H, Ergul A, Figueroa R, Kabuloglu EK, Osborne C, Rowe J, Tattersall E, Leslie A, Xu J, Baek J, Cramer GR, Cushman JC, Cook DR: Characterizing the grape transcriptome. Analysis of expressed sequence tags from multiple Vitis species and development of a compendium of gene expression during berry development. Plant Physiol 2005, I39:574-597.

16. Pacey-Miller T, Scott K, Ablett E, Tingey S, Ching A, Henry R: Genes associated with the end of dormancy in grapes. Funct Integr Genomics 2003, 3:144-152.

17. Espinoza C, Vega A, Medina C, Schlauch K, Cramer G, Arce-Johnson $P$ : Gene expression associated with compatible viral diseases in grapevine cultivars. Funct Integr Genomics 2006. DOI. 10.1007/ s10142-006-0031-6

18. Gomes E, Sagot E, Gaillard C, Laquitaine L, Poinssot B, Sanejouand YH, Delrot S, Coutos-Thevenot P: Nonspecific lipid-transfer protein genes expression in grape (Vitis sp.) cells in response to fungal elicitor treatments. Mol Plant Microbe Interact 2003, 16:456-464.

19. Hain R, Reif HJ, Krause E, Langebartels R, Kindl H, Vornam B, Wiese W, Schmelzer E, Schreier PH, Stocker RH, et al.: Disease resistance results from foreign phytoalexin expression in a novel plant. Nature 1993, 361:153-156.

20. Vitis Expression database [http://cropdisease.ars.usda.gov/ fruit tree/]

21. Gachomo EW, Shonukan OO, Kotchoni SO: The molecular initiation and subsequent acquisition of disease resistance in plants. African J Biotechnol 2003, 2:26-32.

22. Jantasuriyarat C, Gowda M, Haller K, Hatfield J, Lu G, Stahlberg E, Zhou B, Li H, Kim H, Yu Y, Dean RA, Wing RA, Soderlund C, Wang GL: Large-scale identification of expressed sequence tags involved in rice and rice blast fungus interaction. Plant Physio 2005, 138:105-115.

23. Fry SC, Smith RC, Renwick KF, Martin DJ, Hodge SK, Matthews KJ: Xyloglucan endotransglycosylase, a new wall-loosening enzyme activity from plants. Biochem J 1992, 282:82 I-828.

24. Nishitani K: Endo-xyloglucan transferase, a new class of transferase in cell wall construction. J Plant Res 1995, I08: | 37- I 48.

25. Xu W, Campbell P, Vargheese AK, Braam J: The Arabidopsis XET. related gene family: environmental and hormonal regulation of expression. Plant / 1996, 9:879-889.

26. Showalter AM: Structure and function of plant cell wall proteins. Plant Cell 1993, 5:9-23.

27. Bray EA: Genes commonly regulated by water-deficit stress in Arabidopsis thaliana. J Exp Bot 2004, 55:2332-2341.

28. Jacobs AK, Dry IB, Robinson SP: Induction of different pathogenesis-related CDNAs in grapevine infected with powdery mildew and treated with ethephon. Plant Pathol 1999, 48:325-336.

29. Kortekamp A: Expression analysis of defense-related genes in grapevine leaves after inoculation with a host and a non-host pathogen. Plant Physiol Biochem 2006, 44:58-67.

30. Caliskan M: Germin, an oxalate oxidase, has a function in many aspects of plant life. Turk J Biol 2000, 24:717-724.

31. Smalle J, Vierstra RD: The ubiquitin 26S proteasome proteolytic pathway. Annu Rev Plant Biol 2004, 55:555-590.

32. Mahalingam R, Gomez-Buitrago A, Eckardt N, Shah N, Guevara-Garcia A, Day P, Raina R, Fedoroff NV: Characterizing the stress/ defense transcriptome of Arabidopsis. Genome Biol 2003, 4:R20.

33. Rairdan G], Donofrio NM, Delaney TP: Salicylic acid and NIMI/ NPRI-independent gene induction by incompatible Peronospora parasitica in arabidopsis. Molec Plant Microbe Interact 200I, 14: $1235-1246$.

34. Otovis $L J r$ : Antibacterial peptides isolated from insects. J Pept Sci 2000, 6:497-5II.

35. Davis MJ, French W], Schaad NW: Axenic culture of the bacteria associated with phony disease of peach and plum leaf scald. Curr Microbiol I98I, 6:309-3I4.

36. Lewinsohn $E$, Steele CL, Croteau R: Simple isolation of functional RNA from woody stems of gymnosperms. Plant Mol Bio Rep 1994, I 2:20-25.

37. Wang SX, Hunter W, Plant A: Isolation and purification of functional total RNA from woody branches and needles of sitka and white spruce. Bio Techniq 2000, 28:292-296.

38. Link to High Throughput - Gene Ontology - Functional Annotation (HT-GO-FAT) site [http://199.133.147.108/ht-gofat.htm]

39. Gene Ontology website [http://www.geneontology.org/ GO.downloads.shtml] 